\title{
Phenomenological two-stream instability model in the nonlinear electron regime
}

\author{
Paul J. Channell* \\ MS-H817 Los Alamos National Laboratory, Los Alamos, New Mexico 87545
}

(Received 29 August 2002; published 4 November 2002)

\begin{abstract}
We consider the $e-p$ instability for long proton bunches in storage rings. We give a simplified treatment of the centroid model for the linear instability for both unbunched and bunched beams. We point out the very large resulting electron oscillation amplitudes which mean that the electrons very quickly become nonlinear. We then propose a phenomenological theory of the instability in the nonlinear electron regime and show that the resulting model gives a slower growth and different form of the proton oscillations than in the linear theory. We then propose a technique that might eliminate the long term secular growth of the proton oscillations.
\end{abstract}

DOI: 10.1103/PhysRevSTAB.5.114401

PACS numbers: 29.27.-a

\section{INTRODUCTION}

The Proton Storage Ring (PSR) at Los Alamos has been troubled for some time by a violent instability that, when threshold is reached, causes the loss of a large fraction of the stored proton beam, see Refs. [1-14]. There are a number of other high intensity proton rings being considered that may be subject to the same instability, namely, the accumulator ring for the Spallation Neutron Source (SNS) and proton rings for muon production for neutrino sources and muon colliders. A great deal of work has been done attempting to identify the particular instability involved and in finding techniques to eliminate it. Linear theories of a two-stream instability give good agreement with some aspects of the observations, notably the dependence of the threshold on momentum spread, see [1-3]. Most evidence at the moment indicates that the instability probably is a two-stream instability of the proton beam with background electrons, though the detailed source of the electrons has not been definitively identified and many puzzles about the details of the theory and about the number, multiplication, and loss of the electrons remain.

Note that electron cloud effects have been observed in positron rings at B factories at SLAC and KEK and at the Proton Synchrotron and Super Proton Synchrotron at CERN; see Cappi et al. [15], Ohmi et al. [16], and Ohmi and Zimmermann [17], and references contained therein for a discussion of observations and theoretical models. In the B factories the result of the instability is a dilution of the beam emittance and a resulting decrease in the luminosity. In both B factories and at CERN there are many short bunches and both the source of the electrons and the effect of the instability are markedly different from the case in the PSR, SNS, and other proposed machines with long proton bunches. We do not expect that the treatment of the instability given in this paper will be relevant to those machines.

In this paper we will construct a phenomenological nonlinear theory of the two-stream instability motivated by simple linear theory, by the observations of the PSR instability by Macek and collaborators, see Refs. [8-14], and by the simulations of Wang, see $[18,19]$. Both theory and the simulations of Wang indicate that the electrons should reach saturation in their oscillation amplitudes very quickly (in less than one proton bunch passage), so we will use this assumption as the basis of a theory of the instability in the nonlinear electron regime. Because we do not have a detailed theory of the source and evolution of the electron density we will replace this information with a number of assumptions which are plausible and consistent with observations, but subject to uncertainty and which require further experimental investigations and computer simulations.

Briefly, the picture that emerges is that (i) electrons that are either present near the head of the bunch or that rapidly multiply during bunch passage undergo an $e-p$ instability and (in less than one bunch passage) are driven to nonlinear saturation in the nonlinear space charge fields of the proton beam; (ii) this same instability does little to the protons (they oscillate at a much smaller amplitude than the electrons); (iii) the saturated electrons remain oscillating about the tail of the proton bunch and weakly drive proton beam oscillations in the tail; (iv) as the end of the proton bunch passes most of the electrons go to the wall where they are either lost, produce secondaries, or are reflected; and, (v) as the head of the proton beam comes around on the next turn the process repeats and this repetition goes on until the tail of the proton beam is driven to a large amplitude and is lost on the wall. Possibly the onset of proton beam loss leads to a much larger production of electrons and a sudden catastrophic loss of the remaining beam, but we will not model this process. In essence, what occurs is that a linear instability nonlinearly saturates very quickly and produces a constant amplitude driving term that produces linear, secular growth of the proton beam amplitude on a much slower time scale.

In the next section we will review the simplest $e-p$ instability theory for both unbunched and bunched beams and evaluate the ratio of electron to proton oscillation 
amplitudes, which we find to be large, thus justifying the assertion that the $e-p$ instability drives the electron oscillations to nonlinear saturation. In Sec. III we will use models for the nonlinear saturation of the electron amplitudes based on this simplest theory and on assumptions about the proton beam space charge fields, and we will evaluate the effect of the fields of these nonlinearly oscillating electrons on the proton beam after a long period of time in a simple, inhomogeneous, model and show that a secular growth of the proton beam occurs. We compute the resulting growth time and find that, with reasonable assumptions on the neutralization fraction, the observed growth times can be reproduced. We also comment on the importance of a more elaborate linear theory of this instability. In the final section we discuss the model, the assumptions made in it, its limitations, and the need for refinements of the model and for further study.

\section{DIPOLE MODEL OF THE LINEAR $e-p$ INSTABILITY}

In this section we will present a simple theory of the $e-p$ instability. Our purpose is to establish the basic parameters of the theory and define the notation we use. We will consider only cold proton beams and cold electrons in order to simplify the results. We will also comment on the need for a more realistic linear theory.

The model for the linear theory of the instability in this section that we use is the simplest version of the theory of Keil and Zotter, see [20]; for elaborations and extensions of the Keil-Zotter theory, see [21-24]. We model the proton beam by the beam centroid at each azimuthal position around the ring and the background electrons similarly, i.e., by their centroid position at each azimuth. We assume the proton beam moves at a constant azimuthal velocity around the ring and is subject to a constant transverse focusing force that produces betatron oscillations at the betatron frequency. We only model beam and electron motion in one transverse direction. The protons and electrons are assumed to interact with each other via a force that is linear in the relative displacement of the centroids of the protons and electrons. In the subsequent section we will consider more realistic models that take into account the effect of the nonlinearity of the forces.

\section{A. Unbunched beam models}

We begin by considering an unbunched uniform beam model. The equations of motion for the two centroids are given by

$$
\begin{aligned}
\left(\frac{\partial}{\partial t}+\omega_{0} \frac{\partial}{\partial \theta}\right)^{2} y_{p} & =-\omega_{\beta}^{2} y_{p}+\omega_{p}^{2}\left(y_{e}-y_{p}\right), \\
\frac{\partial^{2} y_{e}}{\partial t^{2}} & =\omega_{e}^{2}\left(y_{p}-y_{e}\right),
\end{aligned}
$$

where $y_{p}(\theta, t)$ is the proton centroid position at an azimuth, $\theta$, around the machine and time, $t, y_{e}(\theta, t)$ is the electron centroid position, $\omega_{0}$ is the proton beam angular revolution frequency in the machine, $\omega_{\beta}$ is the angular betatron frequency of the protons in smooth approximation, and the coupling frequencies $\omega_{p}$ and $\omega_{e}$ are given by

$$
\begin{gathered}
\omega_{e}^{2}=\frac{2 N_{p} r_{e} c^{2}}{\pi b(a+b) R}, \\
\omega_{p}^{2}=\left(\frac{f m_{e}}{\gamma m_{p}}\right) \omega_{e}^{2},
\end{gathered}
$$

with $N_{p}$ the number of protons in the machine, $r_{e}$ the classical electron radius, $c$ the velocity of light, $\gamma$ the relativistic factor of the proton beam, $a$ and $b$ the transverse sizes of the proton beam, $f$ the neutralization fraction of electrons, and $R$ the effective radius of the ring. Note that the interspecies force is assumed to depend linearly on the distance between the beam centroids; this is approximately correct for small amplitudes of oscillation, but clearly fails at larger oscillation amplitudes. Note also that we are assuming a constant azimuthal electron density.

The above model is overly simplified but will serve to illustrate the main points of the linear analysis relevant to the present discussion. If we assume that the perturbations have a dependence on time and angle proportional to $e^{-i \omega t+i n \theta}$, then the equations become

$$
\begin{gathered}
{\left[-\left(\omega-n \omega_{0}\right)^{2}+\omega_{\beta}^{2}+\omega_{p}^{2}\right] y_{p}=\omega_{p}^{2} y_{e},} \\
\left(\omega_{e}^{2}-\omega^{2}\right) y_{e}=\omega_{e}^{2} y_{p} .
\end{gathered}
$$

The dispersion equation results from the simultaneous solubility condition for these two equations and is

$$
1=\frac{\omega_{p}^{2} \omega_{e}^{2}}{\left(\omega^{2}-\omega_{e}^{2}\right)\left[\left(\omega-n \omega_{0}\right)^{2}-\omega_{\beta}^{2}-\omega_{p}^{2}\right]}
$$

This is a quartic equation for the frequency. Note that the ratio of electron to proton oscillation amplitudes is given by

$$
\frac{y_{e}}{y_{p}}=\frac{\omega_{e}^{2}}{\omega_{e}^{2}-\omega^{2}} .
$$

In the absence of coupling, the dispersion relation has the solutions $\omega= \pm \omega_{e}$ and $\omega=n \omega_{0} \pm \sqrt{\omega_{\beta}^{2}+\omega_{p}^{2}}$. Instability results when two of these modes almost overlap. Let us define the two frequencies

$$
\begin{aligned}
& \omega_{n+}=n \omega_{0}+\sqrt{\omega_{\beta}^{2}+\omega_{p}^{2}}, \\
& \omega_{n-}=n \omega_{0}-\sqrt{\omega_{\beta}^{2}+\omega_{p}^{2}} .
\end{aligned}
$$

The dispersion relation can then be written as 


$$
1=\frac{\alpha_{1}}{\omega+\omega_{e}}+\frac{\alpha_{2}}{\omega-\omega_{e}}+\frac{\alpha_{3}}{\omega-\omega_{n+}}+\frac{\alpha_{4}}{\omega-\omega_{n-}},
$$

where

$$
\begin{gathered}
\alpha_{1}=\frac{-\omega_{p}^{2} \omega_{e}^{2}}{2 \omega_{e}\left(\omega_{e}+\omega_{n+}\right)\left(\omega_{e}+\omega_{n-}\right)}, \\
\alpha_{2}=\frac{\omega_{p}^{2} \omega_{e}^{2}}{2 \omega_{e}\left(\omega_{e}-\omega_{n+}\right)\left(\omega_{e}-\omega_{n-}\right)}, \\
\alpha_{3}=\frac{\omega_{p}^{2} \omega_{e}^{2}}{\left(\omega_{n+}+\omega_{e}\right)\left(\omega_{n+}-\omega_{e}\right)\left(\omega_{n+}-\omega_{n-}\right)}, \\
\alpha_{4}=\frac{\omega_{p}^{2} \omega_{e}^{2}}{\left(\omega_{n-}+\omega_{e}\right)\left(\omega_{n-}-\omega_{e}\right)\left(\omega_{n-}-\omega_{n+}\right)} .
\end{gathered}
$$

The dispersion relation is usually dominated by the two nearest poles, so one can approximate the other terms as constants; for example, if we look at values of $n$ such that $\omega_{e} \approx \omega_{n-}$ we find the dispersion relation is approximately

$$
1=\frac{\alpha_{2}}{\omega-\omega_{e}}+\frac{\alpha_{4}}{\omega-\omega_{n-}}+\epsilon
$$

where $\epsilon$ is small. This dispersion relation is quadratic and the solutions are

$$
\omega=\frac{\omega_{e}+\omega_{n-}+\bar{\alpha}_{2}+\bar{\alpha}_{4}}{2} \pm \frac{1}{2} \sqrt{\Gamma}
$$

where the radical is

$$
\begin{aligned}
\Gamma= & \left(\omega_{e}+\omega_{n-}+\bar{\alpha}_{2}+\bar{\alpha}_{4}\right)^{2} \\
& -4\left(\omega_{e} \omega_{n-}+\bar{\alpha}_{2} \omega_{n-}+\bar{\alpha}_{4} \omega_{e}\right)
\end{aligned}
$$

and where

$$
\bar{\alpha}_{2}=\frac{\alpha_{2}}{1-\epsilon}
$$

and

$$
\bar{\alpha}_{4}=\frac{\alpha_{4}}{1-\epsilon} .
$$

The radical can be simplified to

$$
\begin{aligned}
\Gamma= & \left(\omega_{e}-\omega_{n-}\right)^{2}+\left(\bar{\alpha}_{2}+\bar{\alpha}_{4}\right)^{2} \\
& +2\left(\bar{\alpha}_{2}-\bar{\alpha}_{4}\right)\left(\omega_{e}-\omega_{n-}\right) .
\end{aligned}
$$

Plugging in the definitions of $\bar{\alpha}_{2}$ and $\bar{\alpha}_{4}$ we find that the radical becomes

$$
\Gamma=\left(\omega_{e}-\omega_{n-}\right)^{2}+\left(\bar{\alpha}_{2}+\bar{\alpha}_{4}\right)^{2}-\frac{\omega_{p}^{2} \omega_{e}\left(\omega_{n-}^{2}+2 \omega_{e}^{2}+\omega_{e} \omega_{n-}-3 \omega_{e} \omega_{n+}-\omega_{n-} \omega_{n+}\right)}{(1-\epsilon) \sqrt{\omega_{\beta}^{2}+\omega_{p}^{2}}\left(\omega_{e}-\omega_{n+}\right)\left(\omega_{e}+\omega_{n-}\right)}
$$

We have instability when $\Gamma<0$. Let us write the solution to the dispersion equation as

$$
\frac{\omega}{\omega_{e}}=\frac{\omega_{e}+\omega_{n-}+\bar{\alpha}_{2}+\bar{\alpha}_{4}}{2 \omega_{e}} \pm \frac{1}{2} \sqrt{\tilde{\Gamma}},
$$

where $\tilde{\Gamma}=\Gamma / \omega_{e}^{2}$. Let us look at an example typical of the PSR; let us take

$$
\begin{gathered}
a=b=1.8 \mathrm{~cm}, \\
\omega_{0}=2 \pi \times 2.8 \mathrm{MHz}, \\
\omega_{\beta}=2.16 \times \omega_{0}, \\
2 \pi R=89 \mathrm{~m},
\end{gathered}
$$

and we find, for various particle numbers (multiplied by $10^{13}$ ) and neutralization fractions, the unstable modes shown in Table I.

The mode frequency scales roughly as the square root of the particle number and the amplitude ratio $\left(y_{e} / y_{p}\right)$ scales roughly as the inverse square root of the neutralization fraction. In all cases the value of $\epsilon$ was small, a few per cent or less. Since in this case we are using an unbunched beam theory, the particle number we should use is roughly the true particle number divided by the bunching factor and thus is about $5 \times 10^{13}$ for conditions where the instability is observed in the PSR. In this simplified model the growth rates are quite high; growth occurs in much less than one turn (the revolution frequency is about $2.8 \mathrm{MHz}$ ). Observed growth times in the PSR range from tens to hundreds of turns; thus one should not give much credence to the above absolute growth rates though the scalings are probably about right. A more elaborate linear theory including various Landau damping effects, see [25-29], decreases the growth rates to more realistic values. Also note that the amplitude ratio is about 20 or greater in these cases so that the electron oscillation amplitude is much larger than the proton oscillation amplitude; a proton oscillation of $1 \mathrm{~mm}$ gives an electron oscillation of $2 \mathrm{~cm}$ or more which is a large enough amplitude that the assumption about the linearity of the force of the protons on the electrons is incorrect due to the decreasing proton beam density at those amplitudes. A proton oscillation amplitude that can lead to loss of the proton beam (probably about $1 \mathrm{~cm}$ or more) would require, in the linear theory, electron oscillation amplitudes of $20 \mathrm{~cm}$ or more, which is well outside the vacuum pipe. Thus, the oscillation amplitudes seem to be at odds with the idea that the instability remains a simple linear two-stream instability for the duration of the observations. On the other hand, the dependence of the threshold for the instability on the momentum spread (the Landau damping) seems to be in agreement with the observations. 
TABLE I. Unstable modes for the two-stream instability for an unbunched beam for the PSR.

\begin{tabular}{cccccc}
\hline \hline Number of particles $\times 10^{-13}$ & Neutralization & Mode & $\begin{array}{c}\text { Growth } \\
(\mathrm{MHz})\end{array}$ & $\begin{array}{c}\text { Frequency } \\
(\mathrm{MHz})\end{array}$ & $y_{e} / y_{p}$ \\
\hline 1 & 0.05 & 26 & 3.76 & 66.7 & 55.7 \\
1 & 0.20 & 26 & 7.51 & 66.7 & 27.9 \\
3 & 0.05 & 43 & 8.01 & 114.9 & 40.9 \\
3 & 0.05 & 44 & 6.48 & 116.3 & 44.3 \\
3 & 0.20 & 42 & 13.9 & 113.3 & 18.7 \\
3 & 0.20 & 43 & 16.9 & 114.8 & 20.7 \\
3 & 0.20 & 44 & 15.6 & 116.2 & 22.4 \\
3 & 0.20 & 45 & 7.52 & 117.6 & 23.7 \\
5 & 0.05 & 54 & 6.12 & 147.1 & 32.4 \\
5 & 0.05 & 55 & 12.3 & 148.5 & 36.1 \\
5 & 0.05 & 56 & 11.0 & 149.9 & 39.1 \\
5 & 0.20 & 54 & 23.5 & 146.7 & 16.8 \\
5 & 0.20 & 55 & 24.6 & 148.2 & 18.5 \\
5 & 0.20 & 56 & 23.2 & 149.7 & 19.9 \\
5 & 0.20 & 57 & 18.3 & 151.1 & 21.1 \\
\hline \hline
\end{tabular}

One of our purposes in this paper is to reconcile these facts. In particular, we will find that the linear theory is very important, it is the starting point and driver for all subsequent effects, and thus it is important to do a more careful analysis of the thresholds and growth rates than was done in this section; see [25-29].

\section{B. Bunched beam models}

To determine the effect of finite bunch length and nonuniform density on the ratio of electron to proton oscillation amplitudes we consider a more general class of models. See Refs. [30,31], for a description of the instability for bunched beams in the time domain rather than the frequency domain. In this subsection we will work in the beam frame of reference because the finite proton beam is easiest to describe in that frame. We again use a centroid model for both the proton beam and the background electrons. The force between the two centroids is assumed to be linear in their relative displacement. The equations of motion for the two centroids in this frame are thus given by

$$
\begin{gathered}
\frac{\partial^{2} y_{p}}{\partial t^{2}}=-\omega_{\beta}^{2} y_{p}+\omega_{p}^{2}\left(y_{e}-y_{p}\right), \\
\left(\frac{\partial}{\partial t}-\omega_{0} \frac{\partial}{\partial \theta}\right)^{2} y_{e}=\omega_{e}^{2}\left(y_{p}-y_{e}\right),
\end{gathered}
$$

where $y_{p}(\theta, t)$ is the proton centroid position at an azimuth, $\theta$, around the machine and time, $t, y_{e}(\theta, t)$ is the electron centroid position, and the coupling frequencies $\omega_{p}$ and $\omega_{e}$ are now given by

$$
\omega_{e}^{2}=\frac{4 \lambda_{p}(\theta) r_{e} c^{2}}{b(a+b)}
$$

$$
\omega_{p}^{2}=\left(\frac{f m_{e}}{\gamma m_{p}}\right) \omega_{e}^{2},
$$

with $\lambda_{p}(\theta)$ the line density of protons in the machine. Note that the interspecies force is assumed to depend linearly on the distance between the beam centroids; this is approximately correct for small amplitudes of oscillation, but clearly fails at larger oscillation amplitudes. Note also that we are assuming that the electron density has the same azimuthal profile as the proton beam; this is probably not realistic given the complex creation, interaction, and loss processes that the electrons undergo and which was alluded to before, but is necessary to get an analytically tractable model. We thus expect that the details of mode shape and the growth rates are unlikely to be accurate, but expect that general features such as the rough magnitude of the ratio of the electron to proton oscillation amplitudes may carry over to the real situation.

We now Fourier transform in time, $t$, but not in angle, $\theta$; we find from Eq. (18) that

$$
y_{e}=\frac{\omega_{\beta}^{2}+\omega_{p}^{2}-\omega^{2}}{\omega_{p}^{2}} y_{p} .
$$

Note that if $\omega_{p}^{2}$ gets small then, locally, the ratio $y_{e} / y_{p}$ gets large. Substituting this into Eq. (19) and Fourier transforming in time we find

$$
\begin{gathered}
\omega_{0}^{2} \frac{\partial^{2} y_{p}}{\partial \theta^{2}}+2 i \omega \omega_{0} \frac{\partial y_{p}}{\partial \theta}+ \\
\left(\omega_{e}^{2}-\omega^{2}-\frac{\omega_{e}^{2} \omega_{p}^{2}}{\omega_{\beta}^{2}+\omega_{p}^{2}-\omega^{2}}\right) y_{p}=0 .
\end{gathered}
$$

If we let 


$$
y_{p}=h e^{-\left[(i \omega \theta) /\left(\omega_{0}\right)\right]},
$$

then we find that $h$ satisfies

$$
\frac{\partial^{2} h}{\partial \theta^{2}}+\left(\frac{\omega_{e}^{2}}{\omega_{0}^{2}}\right)\left(1+\frac{\omega_{p}^{2}}{\omega^{2}-\omega_{\beta}^{2}-\omega_{p}^{2}}\right) h=0 .
$$

\section{Particular bunched beam models}

An exactly soluble model results if we take the proton beam density to be constant for $-\theta_{1} \leq \theta \leq \theta_{1}$ and zero outside this range. Letting

$$
\kappa^{2} \equiv\left(\frac{\omega_{e}^{2}}{\omega_{0}^{2}}\right)\left(1+\frac{\omega_{p}^{2}}{\omega^{2}-\omega_{\beta}^{2}-\omega_{p}^{2}}\right),
$$

Eq. (25) becomes

$$
\frac{\partial^{2} h}{\partial \theta^{2}}+\kappa^{2} h=0
$$

with the solution

$$
h=a e^{ \pm i \kappa \theta},
$$

with $a$ a constant. The solution for $y_{p}$ is then

$$
y_{p}=a e^{ \pm i \kappa \theta-\left[(i \omega \theta) /\left(\omega_{0}\right)\right]},
$$

and the dispersion equation becomes

$$
2\left( \pm \kappa-\frac{\omega}{\omega_{0}}\right) \theta_{1}=2 n \pi, \quad n=1,2,3, \ldots .
$$

We require that the perturbation goes to zero at the bunch ends, which accounts for the integral number of wavelengths. This can be rewritten as

$$
\pm \kappa=\frac{n \pi}{\theta_{1}}+\frac{\omega}{\omega_{0}} \text {. }
$$

Squaring and using Eq. (26) we find

$$
\left(\frac{\omega_{e}^{2}}{\omega_{0}^{2}}\right)\left(1+\frac{\omega_{p}^{2}}{\omega^{2}-\omega_{\beta}^{2}-\omega_{p}^{2}}\right)=\left(\frac{n \pi}{\theta_{1}}+\frac{\omega}{\omega_{0}}\right)^{2} .
$$

This is a quartic equation for $\omega$ and reduces to the usual unbunched beam dispersion relation when $\theta_{1}=\pi$.

The total number of particles in the ring in this case is

$$
N=2 R \lambda \theta_{1},
$$

where $R$ is the effective machine radius. We thus see from Eq. (20) that $\omega_{e}^{2}$ can be rewritten as

$$
\omega_{e}^{2}=\frac{2 N r_{e} c^{2}}{R \theta_{1} b(a+b)} .
$$

For a general density distribution the differential equation is not usually exactly soluble. However, it is possible to find an approximate dispersion relation using the
JWKB technique. Thus let

$$
h=A(\theta) e^{i S(\theta)},
$$

where we now have two unknown functions $A(\theta), S(\theta)$ to solve for and where we assume that the derivatives of $A(\theta), S(\theta)$ are small compared to their values. Plugging this into Eq. (25) we get

$$
A^{\prime \prime}+2 i A^{\prime} S^{\prime}+i S^{\prime \prime} A+\frac{\omega_{e}^{2}}{\omega_{0}^{2}}\left(1+\frac{\omega_{p}^{2}}{\omega^{2}-\omega_{\beta}^{2}-\omega_{p}^{2}}\right) A=0 .
$$

Because we have two unknown functions we can choose to satisfy the equation in various ways. It is convenient to take

$$
2 A^{\prime} S^{\prime}+S^{\prime \prime} A=0
$$

and

$$
A^{\prime \prime}-S^{\prime 2} A+\frac{\omega_{e}^{2}}{\omega_{0}^{2}}\left(1+\frac{\omega_{p}^{2}}{\omega^{2}-\omega_{\beta}^{2}-\omega_{p}^{2}}\right) A=0
$$

In Eq. (38) we drop the $A^{\prime \prime}$ term to get

$$
S^{\prime 2}=\frac{\omega_{e}^{2}}{\omega_{0}^{2}}\left(1+\frac{\omega_{p}^{2}}{\omega^{2}-\omega_{\beta}^{2}-\omega_{p}^{2}}\right)
$$

which can be integrated immediately to give

$$
S(\theta)=\int_{-\theta_{1}}^{\theta} \frac{\omega_{e}\left(\theta^{\prime}\right)}{\omega_{0}}\left(1+\frac{\omega_{p}^{2}\left(\theta^{\prime}\right)}{\omega^{2}-\omega_{\beta}^{2}-\omega_{p}^{2}\left(\theta^{\prime}\right)}\right)^{1 / 2} d \theta^{\prime} .
$$

We can then find $A$ from Eq. (37) as

$$
A=\frac{1}{\sqrt{S^{\prime}}}
$$

The solution for $y_{p}$ is then

$$
y_{p}(\theta)=\frac{1}{\sqrt{S^{\prime}(\theta)}} e^{i S(\theta)-i\left(\omega \theta / \omega_{0}\right)} .
$$

The dispersion relation then becomes

$$
\int_{-\theta_{1}}^{\theta_{1}} \frac{\omega_{e}\left(\theta^{\prime}\right)}{\omega_{0}}\left(1+\frac{\omega_{p}^{2}\left(\theta^{\prime}\right)}{\omega^{2}-\omega_{\beta}^{2}-\omega_{p}^{2}\left(\theta^{\prime}\right)}\right)^{1 / 2} d \theta^{\prime}-\frac{2 \omega \theta_{1}}{\omega_{0}}=2 n \pi, \quad n=1,2,3, \ldots
$$

Again requiring the perturbation to vanish at the bunch ends gives rise to the necessity for an integral number of nodes. 
In general the integral in Eq. (43) is difficult to do analytically, but it is simplified considerably if we realize that in most cases

$$
\left|\omega^{2}\right| \gg \omega_{\beta}^{2} \gg \omega_{p}^{2}
$$

so that Eq. (43) can be approximately written as

$$
\int_{-\theta_{1}}^{\theta_{1}} \frac{\omega_{e}\left(\theta^{\prime}\right)}{\omega_{0}}\left(1+\frac{\omega_{p}^{2}\left(\theta^{\prime}\right)}{2\left(\omega^{2}-\omega_{\beta}^{2}\right)}\right) d \theta^{\prime}-\frac{2 \omega \theta_{1}}{\omega_{0}}=2 n \pi, \quad n=1,2,3, \ldots
$$

If we now take a parabolic density profile for the beam, i.e.,

$$
\lambda(\theta)=\lambda_{0}\left(1-\frac{\theta^{2}}{\theta_{1}^{2}}\right), \quad-\theta_{1} \leq \theta \leq \theta_{1}
$$

then

$$
\omega_{e}(\theta)=\omega_{e 0} \sqrt{1-\frac{\theta^{2}}{\theta_{1}^{2}}}
$$

and

$$
\omega_{p}(\theta)=\omega_{p 0} \sqrt{1-\frac{\theta^{2}}{\theta_{1}^{2}}}
$$

The dispersion relation [Eq. (45)] becomes

$$
\frac{\pi \theta_{1} \omega_{e 0}}{2 \omega_{0}}\left(1+\frac{3 \omega_{p 0}^{2}}{4\left(\omega^{2}-\omega_{\beta}^{2}\right)}\right)-\frac{2 \omega \theta_{1}}{\omega_{0}}=2 n \pi, \quad n=1,2,3, \ldots
$$

Note that the solution, $y_{p}(\theta)$ has a linear zero at $\pm \theta_{1}$ from the exponential, but a fourth root singularity at those points due to the coefficient, so overall it vanishes like the $3 / 4$ power at $\pm \theta_{1}$. In the solution $y_{p}(\theta)$ the coefficient is symmetric in $\theta$ whereas the expression in the exponent is odd in $\theta$. Thus, the magnitude of the solution tends to grow toward both the front and the back of the bunch until near the bunch ends where it vanishes. With a more realistic model for the electron density, including the asymmetric accumulation of electrons during bunch passage, this front-back symmetry of the magnitude is likely to be broken.

\section{Numerical results}

We have solved the dispersion relations of Eqs. (32) and (49) with the parameters

$$
\begin{gathered}
a=b=1.8 \mathrm{~cm}, \\
\omega_{0}=2 \pi \times 2.8 \mathrm{MHz}, \quad \omega_{\beta}=2.16 \times \omega_{0}, \\
2 \pi R=89 \mathrm{~m},
\end{gathered}
$$

as before and with different numbers of protons in the ring (times $10^{13}$ ). With a neutralization fraction of $f=0.2$ the results are shown in Table II.

As one can see, the growth rates are substantial in this simple model, though again, more sophisticated models with Landau damping will give smaller growth rates. In particular one should note that the central ratio of the electron oscillation amplitude to the proton oscillation amplitude is large (roughly 20 or greater for the cases shown here).

For a neutralization fraction of $f=0.02$ we get the results shown in Table III.

As can be seen, in this case the growth rates are smaller, though still larger than those observed, but the central ratio of electron oscillation amplitude to proton oscillation amplitude is larger.

\section{Stability summary}

We have seen in this section that the ratio of electron to proton oscillation amplitudes is large, ranging from about 20 for higher neutralization fractions to about 80 for lower neutralization fractions. For finite length nonuniform bunches, this ratio tends to be larger toward the end of the bunches as can be seen from Eq. (22). Though the growth rates derived in this section are unlikely to be realistic, the large ratio of electron to proton oscillation amplitudes found and its scaling almost certainly persists in better models, as it is basically just due to the mass ratio of the electrons and protons. Thus, even very small proton oscillation amplitudes will result in electron oscillations that are in the nonlinear regime. 
TABLE II. Unstable modes for the two-stream instability for a bunched beam for the PSR with a neutralization fraction of 0.2 .

\begin{tabular}{|c|c|c|c|c|}
\hline Type & $\begin{array}{c}\text { Number of } \\
\text { particles } \times 10^{-13}\end{array}$ & $\begin{array}{c}\text { Angular } \\
\text { extent }\end{array}$ & $\begin{array}{l}\text { Growth } \\
(\mathrm{MHz})\end{array}$ & $\begin{array}{c}y_{e} / y_{p} \\
\text { (Bunch center) }\end{array}$ \\
\hline Constant & 1 & 1 & 5.331 & 39.316 \\
\hline Constant & 1 & 0.8 & 6.281 & 36.972 \\
\hline Constant & 1 & 0.6 & 6.861 & 36.118 \\
\hline Constant & 1 & 0.4 & 10.362 & 31.982 \\
\hline Constant & 3 & 1 & 11.541 & 28.731 \\
\hline Constant & 3 & 0.8 & 13.371 & 29.916 \\
\hline Constant & 3 & 0.6 & 17.065 & 25.633 \\
\hline Constant & 3 & 0.4 & 22.871 & 24.893 \\
\hline Constant & 5 & 1 & 17.065 & 25.633 \\
\hline Constant & 5 & 0.8 & 20.056 & 24.383 \\
\hline Constant & 5 & 0.6 & 24.102 & 21.963 \\
\hline Constant & 5 & 0.4 & 31.491 & 19.668 \\
\hline Parabolic & 1 & 1 & 5.665 & 43.102 \\
\hline Parabolic & 1 & 0.8 & 4.715 & 38.324 \\
\hline Parabolic & 1 & 0.6 & 7.801 & 38.770 \\
\hline Parabolic & 1 & 0.4 & 8.972 & 36.355 \\
\hline Parabolic & 3 & 1 & 12.368 & 31.052 \\
\hline Parabolic & 3 & 0.8 & 14.778 & 32.156 \\
\hline Parabolic & 3 & 0.6 & 18.420 & 27.502 \\
\hline Parabolic & 3 & 0.4 & 24.560 & 24.856 \\
\hline Parabolic & 5 & 1 & 18.772 & 29.307 \\
\hline Parabolic & 5 & 0.8 & 21.991 & 27.533 \\
\hline Parabolic & 5 & 0.6 & 26.038 & 23.753 \\
\hline Parabolic & 5 & 0.4 & 35.010 & 23.146 \\
\hline
\end{tabular}

\section{THE NONLINEAR ELECTRON REGIME}

In this section we attempt to model the interaction of the electrons and protons after the electrons are driven by the proton oscillations at the head of the bunch into the nonlinear regime. We begin by considering the interactions on a single passage of a proton bunch and then iterate the resulting picture to find the long term growth rates of the protons.

\section{A. Single passage of a bunch}

Let us consider the sequence of events as a bunch passes an electron cloud. Electrons that are either left over from the previous passage or are generated at the walls and are drawn in by the bunch are kicked transversely by the offset protons at or near the head of the bunch. The electrons will be kicked to some initial oscillation amplitude which will be significant and this amplitude will grow somewhat due to the two-stream instability. As the electrons oscillate at larger amplitudes their oscillation frequency decreases (due to the nonlinear space charge force of the proton beam) and they fall out of resonance with the two-stream driving mechanism; the
TABLE III. Unstable modes for the two-stream instability for an unbunched beam for the PSR with a neutralization fraction of 0.02 .

\begin{tabular}{ccccc}
\hline \hline Type & $\begin{array}{c}\text { Number of } \\
\text { particles } \times 10^{-13}\end{array}$ & $\begin{array}{c}\text { Angular } \\
\text { extent }\end{array}$ & $\begin{array}{c}\text { Growth } \\
\text { (MHz) }\end{array}$ & $\begin{array}{c}y_{e} / y_{p} \\
\text { (Bunch center) }\end{array}$ \\
\hline Constant & 1 & 1 & 1.689 & 123.950 \\
Constant & 1 & 0.8 & 1.900 & 116.420 \\
Constant & 1 & 0.6 & 0.000 & 0.000 \\
Constant & 1 & 0.4 & 3.132 & 100.150 \\
Constant & 3 & 1 & 0.000 & 0.000 \\
Constant & 3 & 0.8 & 2.669 & 93.190 \\
Constant & 3 & 0.6 & 3.888 & 78.610 \\
Constant & 3 & 0.4 & 7.598 & 75.590 \\
Constant & 5 & 1 & 3.888 & 78.610 \\
Constant & 5 & 0.8 & 5.002 & 74.020 \\
Constant & 5 & 0.6 & 5.487 & 78.590 \\
Constant & 5 & 0.4 & 5.684 & 73.690 \\
Parabolic & 1 & 1 & 1.548 & 135.830 \\
Parabolic & 1 & 0.8 & 0.000 & 0.000 \\
Parabolic & 1 & 0.6 & 0.000 & 0.000 \\
Parabolic & 1 & 0.4 & 0.000 & 0.000 \\
Parabolic & 3 & 1 & 2.129 & 106.170 \\
Parabolic & 3 & 0.8 & 3.870 & 99.860 \\
Parabolic & 3 & 0.6 & 1.390 & 83.640 \\
Parabolic & 3 & 0.4 & 2.569 & 73.420 \\
Parabolic & 5 & 1 & 6.122 & 89.980 \\
Parabolic & 5 & 0.8 & 7.020 & 83.520 \\
Parabolic & 5 & 0.6 & 7.846 & 83.210 \\
Parabolic & 5 & 0.4 & 10.046 & 65.730 \\
\hline \hline
\end{tabular}

instability stops growing. However, the electrons are still oscillating and their mean space charge couples to the proton beam, especially if the large amplitude electron bounce frequency falls onto a betatron sideband of the proton beam. The result is a secular growth of transverse oscillations in the tail of the proton beam on a time scale of many turns. Note that as the electrons drive the tail of the proton beam they actually lose some of their oscillation amplitude and could, if the proton beam were long enough, fall back onto the frequency for the instability; in this paper, for simplicity, we assume this does not happen.

As the tail of the proton bunch passes, almost all electrons go to the walls where some are lost, some create more electrons by secondary emission, and some are reflected; see the works of Blaskiewicz [32,33] and Furman and Pivi [34] for a more detailed discussion and simulations of these processes. At the next passage of the proton bunch the process repeats. Note that the head of the proton beam will, if the linear mode is neutrally stable or unstable, retain its initial oscillations at the same phase from turn to turn and will induce the (assumed) fresh electrons on the next turn to lock in their oscillations at the same phase. 
Of course, if electrons being kicked by the protons were the only process going on near the head of the bunch, then the proton oscillations would be quickly damped as the transverse oscillation energy was fed into the electrons; all subsequent oscillations would die away. In order to sustain the proton oscillations it is necessary that the usual $e-p$ mode be unstable with some positive growth rate sufficient to overcome the damping due to the transverse energy imparted to the electrons. If the $e-p$ mode is unstable then the source that drives the oscillations is ultimately the translational motion of the proton beam, a very large source of free energy. Thus, though the slow growth of the transverse oscillations of the proton bunch is due to nonlinear processes, the onset and persistence of the phenomenon depends on the linear threshold of the $e-p$ instability; it is thus very important to do a much better job of determining instability thresholds than was presented in the previous section; see [25-29].

\section{B. Simple model for proton beam driving}

At the head of the proton bunch the proton oscillation amplitudes remain small for quite some time; we assume that a more sophisticated and realistic variant of the linear theory presented in the second section describes the behavior of the protons and electrons in this portion of the bunch. Toward the end of the proton bunch we assume that the electrons have reached saturation and are oscillating at a constant amplitude; in addition, for simplicity, we assume that this amplitude of oscillation of the electrons is the same from turn to turn and that the fractional neutralization is the same from turn to turn. Clearly these simplifying assumptions can be replaced by other, more realistic assumptions, but the resulting model will serve to illustrate the important features of the theory. We also assume that the electron oscillation phase remains coherent from turn to turn; this is certainly reasonable as the oscillation phase is determined by the oscillation phase of the protons at the head of the bunch.

With these assumptions we can model the motion of the protons in the tail of the bunch by Eq. (1) with the electron motion given as a fixed oscillation, i.e., the equation becomes

$$
y_{p}=\omega_{p}^{2} \tilde{y} \int \operatorname{dah}(a)\left(\frac{e^{-i a t}}{\left(a-\omega_{1}\right)\left(a-\omega_{2}\right)}+\frac{e^{-i \omega_{1} t}}{\left(\omega_{1}-a\right)\left(\omega_{1}-\omega_{2}\right)}+\frac{e^{-i \omega_{2} t}}{\left(\omega_{2}-\omega_{1}\right)\left(\omega_{2}-a\right)}\right)+\text { IVT. }
$$

Using the identity

$$
\frac{1}{\left(a-\omega_{1}\right)\left(a-\omega_{2}\right)}=\frac{1}{\left(\omega_{1}-\omega_{2}\right)\left(a-\omega_{1}\right)}+\frac{1}{\left(\omega_{2}-\omega_{2}\right)\left(a-\omega_{2}\right)},
$$

we can rewite Eq. (62) as

$$
\begin{aligned}
y_{p}=-\omega_{p}^{2} \tilde{y} \int d a h(a) & \left(\frac{e^{-i a t}}{\left(\omega_{1}-\omega_{2}\right)\left(a-\omega_{1}\right)}+\frac{e^{-i a t}}{\left(\omega_{2}-\omega_{1}\right)\left(a-\omega_{2}\right)}+\frac{e^{-i \omega_{1} t}}{\left(\omega_{1}-a\right)\left(\omega_{1}-\omega_{2}\right)}\right. \\
& \left.+\frac{e^{-i \omega_{2} t}}{\left(\omega_{2}-a\right)\left(\omega_{2}-\omega_{1}\right)}\right)+\mathrm{IVT} .
\end{aligned}
$$

where IVT indicates initial value terms. These initial value terms merely lead to persistent oscillations that do not grow in time so we will ignore them. Solving we find

$$
\bar{y}_{p}=\frac{\omega_{p}^{2} \bar{y}_{e}}{\left(s+i n \omega_{0}\right)^{2}+\omega_{\beta}^{2}+\omega_{p}^{2}}+\mathrm{IVT} .
$$

Let

$$
y_{e}=\tilde{y} \int d a e^{i a t} h(a)
$$

If we define

$$
\begin{gathered}
\omega_{1} \equiv n \omega_{0}-\sqrt{\omega_{\beta}^{2}+\omega_{p}^{2}}, \\
\omega_{2} \equiv n \omega_{0}+\sqrt{\omega_{\beta}^{2}+\omega_{p}^{2}},
\end{gathered}
$$

then

$$
\bar{y}_{p}=\omega_{p}^{2} \int d a \frac{h(a)}{(s+i a)\left(s+i \omega_{1}\right)\left(s+i \omega_{2}\right)}+\text { IVT. }
$$

Doing the inverse Laplace transform we find

Then

$$
\bar{y}_{e}=\tilde{y} \int d a \frac{h(a)}{s+i a} .
$$

ent driving amplitudes and frequencies for $y_{e}$. If we assume a dependence on $\theta$ of the form $e^{i n \theta}$, i.e., assume 列

$$
\frac{\partial^{2} y_{p}}{\partial t^{2}}+2 i n \omega_{0} \frac{\partial y_{p}}{\partial t}-n^{2} \omega_{0}^{2} y_{p}+\left(\omega_{\beta}^{2}+\omega_{p}^{2}\right) y_{p}=\omega_{p}^{2} y_{e} .
$$


Combining terms this can be rewritten as

$$
y_{p}=-\omega_{p}^{2} \tilde{y} \int d a h(a)\left(\frac{e^{-i a t}-e^{-i \omega_{1} t}}{\left(\omega_{1}-\omega_{2}\right)\left(a-\omega_{1}\right)}-\frac{e^{-i a t}-e^{-i \omega_{2} t}}{\left(\omega_{1}-\omega_{2}\right)\left(a-\omega_{2}\right)}\right)+\text { IVT. }
$$

Finally, using the expressions for $\omega_{1}$ and $\omega_{2}$ and taking out common factors we get

$$
y_{p}=\frac{-i \omega_{p}^{2} \tilde{y}}{2 \sqrt{\omega_{\beta}^{2}+\omega_{p}^{2}}}\left(\int_{0}^{t} d t^{\prime} e^{i \omega_{1}\left(t^{\prime}-t\right)} \int d a h(a) e^{-i a t^{\prime}}-\int_{0}^{t} d t^{\prime} e^{i \omega_{2}\left(t^{\prime}-t\right)} \int d a h(a) e^{-i a t^{\prime}}\right)+\text { IVT. }
$$

Note that the form of the time dependence of the result depends on the distribution of electron bounce frequencies. In the next subsections we will consider two distributions.

\section{Delta function distribution}

If we take

$$
h(a)=\delta\left(a-\omega_{1}\right)
$$

then we find that

$$
y_{p}=\frac{-i \omega_{p}^{2} \tilde{y} t e^{-i \omega_{1} t}}{2 \sqrt{\omega_{\beta}^{2}+\omega_{p}^{2}}}+\text { oscillatory terms }
$$

i.e., there is a secular growth in time. This is just the usual linear growth in time that results when a linear oscillator is driven on resonance. This is the result we expect when the fast $e-p$ mode is unstable at the frequency $\omega_{1}$ and the electrons all saturate at relatively small amplitudes where the proton space charge has not begun to fall off significantly. We could, of course, also consider driving at the frequency $\omega_{2}$ but there are unlikely to be many electrons oscillating at that frequency as the upper side band is stable.

We can write the magnitude of the secular term as

$$
\left|\frac{y_{p}}{\tilde{y}}\right|=\frac{\bar{\omega}_{p}^{2} t}{2 \sqrt{\omega_{\beta}^{2}+\bar{\omega}_{p}^{2}}} .
$$

This becomes of the order of 1 at a time given by

$$
t=\frac{2 \sqrt{\omega_{\beta}^{2}+\bar{\omega}_{p}^{2}}}{\bar{\omega}_{p}^{2}}
$$

Plugging in the values used in Sec. II we find that

$$
\bar{\omega}_{p}^{2}=\bar{f} \bar{N}_{p} \times 50.182 \times 10^{14} \mathrm{sec}^{-2},
$$

and

$$
\omega_{\beta}^{2}=1.444 \times 10^{15} \mathrm{sec}^{-2},
$$

where $N_{p}=\bar{N}_{p} \times 10^{13}$. The time for the growth of proton beam oscillations is thus

$$
t=\frac{0.88 \sqrt{2.79+\bar{f} \bar{N}_{p}}}{\bar{f} \bar{N}_{p}} 10^{-7} \mathrm{sec} .
$$

If we take $\bar{N}_{p}=2$ (appropriate for the tail of the bunch) and the effective neutralization (electrons resonant with the betatron sideband) to be $\bar{f}=0.01$, then we find $t=7.35 \mu \mathrm{sec}$ for the proton beam oscillations to grow to the size of the small amplitude electron beam oscillations.

\section{Rectangular distribution}

It is likely that as the amplitude of the fast instability grows the electron bounce distribution is not a delta function but has a maximum at $\omega_{1}$ for small amplitude electrons, where it is driven by the instability, and extends downward in frequency for the larger amplitude electrons which have lower frequencies in the nonlinear space charge of the proton beam. Analytically, we have

$$
h(a)=\left[\begin{array}{cc}
\frac{1}{\Delta \omega} & \text { for } \omega_{1}-\Delta \omega \leq a \leq \omega_{1} \\
0 & \text { otherwise }
\end{array}\right] .
$$

We then find that the proton response becomes

$$
\begin{aligned}
y_{p}=\frac{\omega_{p}^{2} \tilde{y} e^{-i \omega_{1} t}}{2 \sqrt{\omega_{\beta}^{2}+\omega_{p}^{2}}} & \left(\int_{0}^{t} d t^{\prime} \frac{\left(1-e^{i \Delta \omega t^{\prime}}\right)}{\Delta \omega t^{\prime}}\right. \\
& \left.-\int_{0}^{t} d t^{\prime} e^{i\left(\omega_{2}-\omega_{1}\right) t^{\prime}} \frac{\left(1-e^{i \Delta \omega t^{\prime}}\right)}{\Delta \omega t^{\prime}}\right)+ \text { IVT. }
\end{aligned}
$$

Unless $\Delta \omega$ is very small then all terms are oscillatory except for one; we can thus write this as

$$
y_{p}=\frac{\omega_{p}^{2} \tilde{y} e^{-i \omega_{1} t}}{2 \Delta \omega \sqrt{\omega_{\beta}^{2}+\omega_{p}^{2}}} \int_{t_{0}}^{t} \frac{d \tau}{\tau}+\text { oscillatory terms }
$$

i.e.,

$$
y_{p}=\frac{\omega_{p}^{2} \tilde{y} e^{-i \omega_{1} t}}{2 \Delta \omega \sqrt{\omega_{\beta}^{2}+\omega_{p}^{2}}} \ln \left(\frac{t}{t_{0}}\right)+\text { oscillatory terms. }
$$

Thus, in this case we find logarithmic growth of the proton amplitudes. If we take absolute magnitudes and solve for the time we find 


$$
t=t_{0} \exp \left(2 \frac{y_{p}}{\tilde{y}} \frac{\Delta \omega \sqrt{\omega_{\beta}^{2}+\omega_{p}^{2}}}{\omega_{p}^{2}}\right) .
$$

It is easy to get reasonable growth times with plausible choices of parameters, but slightly different choices of parameters can result in very different growth times. Of course, the concept of a growth time in this case is not very well defined.

\section{E. Stabilization}

Note that the vital ingredient of this model is that the phase coherence is maintained by the very small oscillations at the head of the proton bunch. This suggests that it may be possible to reduce the effects of the instability if one can drive the head of the bunch on a frequency different from a betatron sideband frequency. This should be somewhat easier to do than feedback stabilization because (i) it does not require one to detect the oscillation, and (ii) one has to kick the beam only a small distance, say, about $0.05 \mathrm{~mm}$ which requires an applied transverse electric field of about $20 \mathrm{kV} / \mathrm{m}$. The net effect of these kicks is cancellation of the proton oscillations in the tail of the bunch (but not the electron oscillations), except for a random diffusion on a much longer time scale. Though the equipment to test this cure does not presently exist on the PSR, it may be installed and tried in a future run.

Once the applied $\mathrm{rf}$ forces are considered the proton oscillations can be carried toward the front of the bunch as the protons rotate in longitudinal phase space. Thus, on the time scale of a quarter of an rf bucket oscillation, we expect that the perturbation will move toward the head of the bunch. It is thus important to stop the growth of the instability on a faster time scale than this.

\section{SUMMARY AND CONCLUSIONS}

The linear models we presented in Sec. II are overly simplified but serve nicely to illustrate the difficulty with the linear theory, namely, the very large electron oscillation amplitudes. The applicability of the threshold derived from the linear theory is due to the fact that the entire process, though it evolves nonlinearly, depends on the linear instability to sustain the small head oscillations and lock in the phase from turn to turn. If the linear mode is suppressed then the whole process does not go. It is thus important to do a better job modeling the linear instability than was done in Sec. II.

Using the picture of large electron oscillation amplitudes given by this model we were able in Sec. III to model the nonlinear electron regime as a simple driving term for the proton on the tail of the bunch and to derive growth times for the protons that seem about right. In particular, an important ingredient of this theory is that the coherent phase of the proton beam driving from turn to turn is carried by the small oscillations at the head of the proton beam and thus may be ameliorated by driving small transverse oscillations at the head of the proton bunch at a frequency off a betatron sideband in order to destroy phase coherence from turn to turn.

The main unanswered questions of this phenomenological theory include the source of the electrons, the actual neutralization conditions, and the detailed description of the time evolution of the perturbations. From the theory given in this paper we see that it is important that there be only enough electrons present for the linear instability to evolve. The number of electrons present and their distribution does affect the functional form of the proton beam growth, but the basic time scale is somewhat insensitive to the details.

Finally, it is likely that a more accurate account of the time evolution than we have given can result only from detailed computer simulations or from elaborate matched asymptotic expansions with different dynamical assumptions in the various regions of the beam.

*Email address: pchannell@lanl.gov

[1] D. Neuffer, E. Colton, G. Swain, H. A. Thiessen, B. Blind, A. Jason, G. Lawrence, R. Shafer, T. Hardek, J. Hurd, R. Macek, and M. Plum, Part. Accel. 23, 133 (1988).

[2] E. P. Colton, D. Neuffer, G. Swain, H. A. Thiessen, A. Lombardi, and T.-S. Wang, in Proceedings of the 1989 Particle Accelerator Conference, Chicago, IL (IEEE, Piscataway, NJ, 1989), Vol. 2, p. 1202.

[3] D. Neuffer and T. Wang, in Proceedings of the Advanced Hadron Facility Accelerator Design Workshop (Los Alamos National Laboratory Report No. LA-11684-C, 1989), p. 794.

[4] T.-S. F. Wang, A. Pisent, and D.V. Neuffer, in Proceedings of the Advanced Hadron Facility Accelerator Design Workshop, 1989 (Ref. [3]), p. 818.

[5] E. Colton, D. Fitzgerald, T. Hardek, R. Macek, M. Plum, H. Thiessen, T. Wang, and D. Neuffer, in Proceedings of the 1991 Particle Accelerator Conference, San Francisco (IEEE, Piscataway, NJ, 1991), Vol. 3, p. 1896.

[6] D. Neuffer, E. Colton, D. Fitzgerald, T. Hardek, R. Hutson, R. Macek, M. Plum, H. Thiessen, and T.-S. Wang, Nucl. Instrum. Methods Phys. Res., Sect. A 321, 1 (1992).

[7] T. Wang, P. J. Channell, R. K. Cooper, D. H. Fitzgerald, T. Hardek, R. Hutson, A. J. Jason, R. J. Macek, M. A. Plum, C. Wilkinson, and E. Colton, in Proceedings of the 1995 Particle Accelerator Conference, Dallas, TX (IEEE, Piscataway, NJ, 1995), Vol. 5, p. 3146.

[8] M. A. Plum, D. H. Fitzgerald, D. Johnson, J. Langenbrunner, R. J. Macek, F. Merrill, P. Morton, B. Prichard, O. Sander, M. Shulze, H. A. Thiessen, T.S. Wang, and C. A. Wilkinson, in Proceedings of the 1997 Particle Accelerator Conference, Vancouver (IEEE, Piscataway, NJ, 1997), p. 1611. 
[9] Proceedings of the Santa Fe Workshop on Electron Effects, edited by T. F. Wang and A. Jason (Los Alamos National Laboratory Report No. LA-UR-98-1601, 1998).

[10] R. J. Macek, A. Browman, D. H. Fitzgerald, R. McCrady, M. Plum, and T. Spickermann, in Proceedings of the ICANS-XV, KEK, Tsukuba, Japan, 2000 (to be published).

[11] Proceedings of the ICFA 8th Beam Dynamics MiniWorkshop on Two-Stream Instabilities in Particle Accelerators and Storage Rings, Santa $\mathrm{Fe}$, New Mexico, 2000. In particular, Session I, http://www. aps.anl.gov/conferences/icfa/two-stream.html.

[12] R. J. Macek et al., in Proceedings of the 2001 Particle Accelerator Conference, Chicago (IEEE Catalog No. 01CH37268), Vol. 1, p. 688.

[13] R. J. Macek et al., in Proceedings of the International Workshop on Two-Stream Instabilities in Particle Accelerator and Storage Rings, 2001, Santa Fe, NM (KEK, Tsukuba-shi, Japan, 2001). In particular, Session I, http://www.aps.anl.gov/conferences/icfa/ two-stream.html

[14] R. J. Macek et al., Los Alamos Report No. LA-13943PR, 2002, p. 50.

[15] R. Cappi, M. Giovannozzi, E. Métral, G. Rumolo, and F. Zimmermann, Phys. Rev. ST Accel. Beams 5, 094401 (2002).

[16] K. Ohmi, F. Zimmermann, and E. Perevdentsev, Phys. Rev. E 65, 016502 (2001).

[17] K. Ohmi and F. Zimmermann, Phys. Rev. Lett. 85, 3821 (2000).

[18] T. Wang, in A Simple Simulation of Electron-Proton Instability, AIP Conf. Proc. No. 496 (AIP, New York, 1999), p. 305.

[19] T. Wang, in Proceedings of the 1995 Particle Accelerator Conference, Dallas, TX (Ref. [7]), Vol. 5, p. 3143.

[20] E. Keil and B. Zotter, CERN Report No. CERN-ISR-TH/ 71-58, 1971.
[21] L. J. Laslett, A. M. Sessler, and D. Möhl, Nucl. Instrum. Methods 121, 517 (1974).

[22] H. Schönauer and B. Zotter, “Electron-Proton Instability in the SPS," CERN Internal Note, 1972.

[23] D. G. Koshkarev and P. R. Zenkevich, Part. Accel. 3, 1 (1972).

[24] H. G. Hereward, CERN Internal Report No. 71-15, 1971.

[25] R. C. Davidson, H. Qin, P. H. Stoltz, and T-S F. Wang, Phys. Rev. ST Accel. Beams 2, 054401 (1999).

[26] R. C. Davidson and Hong Qin, Phys. Lett. A 270, 177 (2000).

[27] R. C. Davidson, H. Qin, W.W. Lee, and T. F. Wang, in Proceedings of the 1999 Particle Accelerator Conference, New York (IEEE, Piscataway, NJ, 1999), Vol. 3, p. 1623.

[28] H. Qin, R. C. Davidson, W.W. Lee, and E. Startsev, in Proceedings of the 2001 Particle Accelerator Conference (Ref. [12]), p. 696.

[29] H. Qin, R. C. Davidson, and W.W. Lee, in Proceedings of the 1999 Particle Accelerator Conference (Ref. [27]), Vol. 3, p. 1628.

[30] T. F. Wang, P. J. Channell, R. J. Macek, and R. C. Davidson, in Proceedings of the 2001 Particle Accelerator Conference (Ref. [12]), Vol. 1, p. 704.

[31] T. F. Wang, Los Alamos National Laboratory Internal Report No. LANSCE1:02-035, 2002.

[32] M. Blaskiewicz, in Proceedings of the 1999 Particle Accelerator Conference, New York (Ref. [27]), pp. 16111613.

[33] M. Blaskiewicz, in The Fast Loss Electron-Proton Instability, AIP Conf. Proc. No. 496 (AIP, New York, 1999) p. 321.

[34] M. A. Furman and M. Pivi, in Proceedings of the 2001 Particle Accelerator Conference (Ref. [12]), p. 707. 